\title{
Revealing the Underestimated Anticancer Effect of Azurin by Mechanical Unfolding
}

Xiangchao Pang, ${ }^{\dagger, \downarrow}$ Caijie Yuan, ${ }^{\dagger}$ Rui Sun,${ }^{\S}$ Kui Wang, ${ }^{\S}$ Bin Tang ${ }^{*}, \beta, \|, \perp$ 'College of Materials Science and Engineering, Central South University of Forestry and Technology, Changsha 410004, PR China

${ }^{\sharp}$ Hunan Province Key Laboratory of Materials Surface \& Interface Science and Technology, Central South University of Forestry and Technology, Changsha 410004, China

${ }^{\S}$ Department of Biomedical Engineering, Southern University of Science and Technology, Shenzhen 518055, China

"Shenzhen Key Laboratory of Cell Microenvironment, Southern University of Science and Technology, Shenzhen 518055, China

${ }^{\perp}$ Guangdong Provincial Key Laboratory of Cell Microenvironment and Disease Research, Southern University of Science and Technology, Shenzhen 518055, China

"Email: tangb@sustech.edu.cn

\section{GB1: the fingerprint domain}

In this study, GB1, the B1 immunoglobulin (IgG)-binding domain of protein G from the surface of certain Staphylococcal and Streptococcal strains ${ }^{1}$, was used as a fingerprint to identify single-molecule stretching events. There are two main reasons to select GB1 as the fingerprint: (1) the structure of GB1 domain is very representative. The GB1 domain is a small $\alpha / \beta$ protein with only 56 amino acid residues and is composed of a four-strand $\beta$ sheet packed against a long $\alpha$ helix ${ }^{2}$. This representative structure can be used to characterize the unfolding behavior of $\alpha$ helix at a very low force. (2) the 
unfolding behaviors (contour length increment $(\Delta \mathrm{Lc})$ and unfolding force) of GB1 domains have been well characterized in many studies ${ }^{3,4}$. It demonstrates that $\Delta \mathrm{Lc}$ is about $18 \mathrm{~nm}$, and the unfolding force is $180 \mathrm{pN}$ at a pulling speed of $400 \mathrm{~nm} / \mathrm{s}$ for GB1. Besides that, GB1 can be also as an internal force caliper for the measurements of the unfolding force for azurin unfolding.

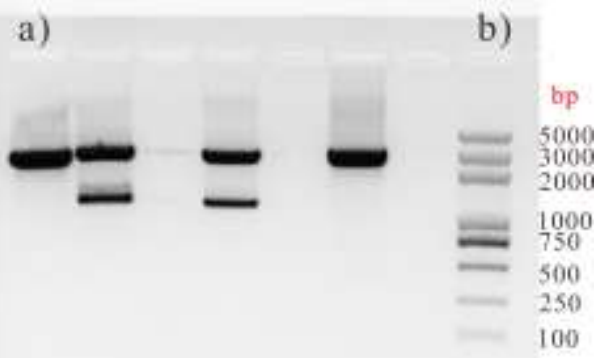

Fig. S1 An agarose gel stained with ethidium bromide showing cDNAs of the $(\text { azurin+GB1) })_{4}($ lane a). Lane b) is the marker.

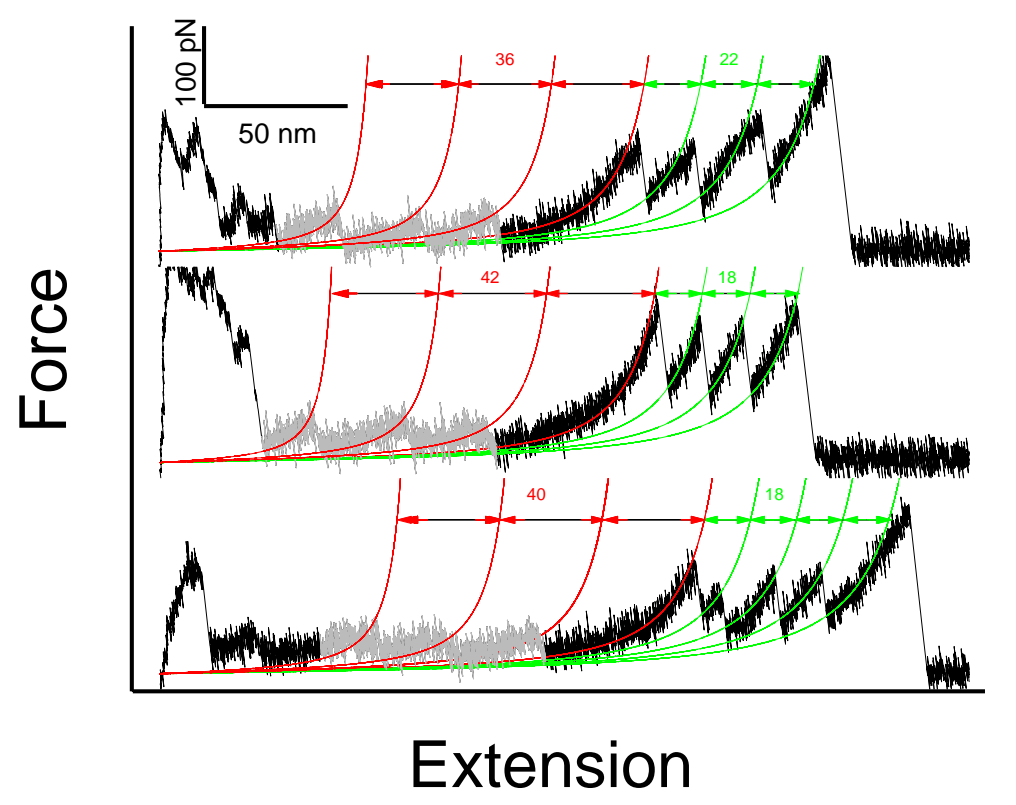

Fig. S2 Some typical force-extension curves of (apo-azurin+GB1) 4 . The unfolding force peaks were fitting using the WLC model. The red lines 
correspond to the fitting curve of apo-azurin. The green lines represent the fitting curves of GB1.

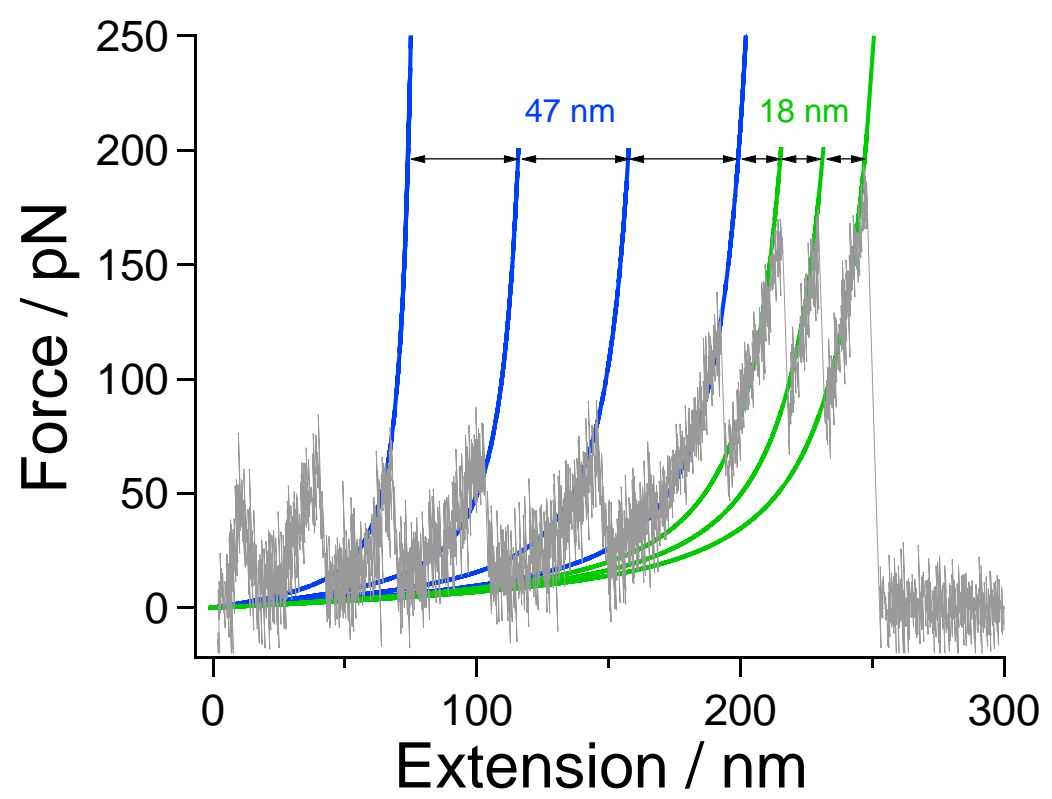

Fig. S3 Typical force-extension curves of (apo-azurin+GB1) ${ }_{4}$ with the presence of DTT (100 mM).

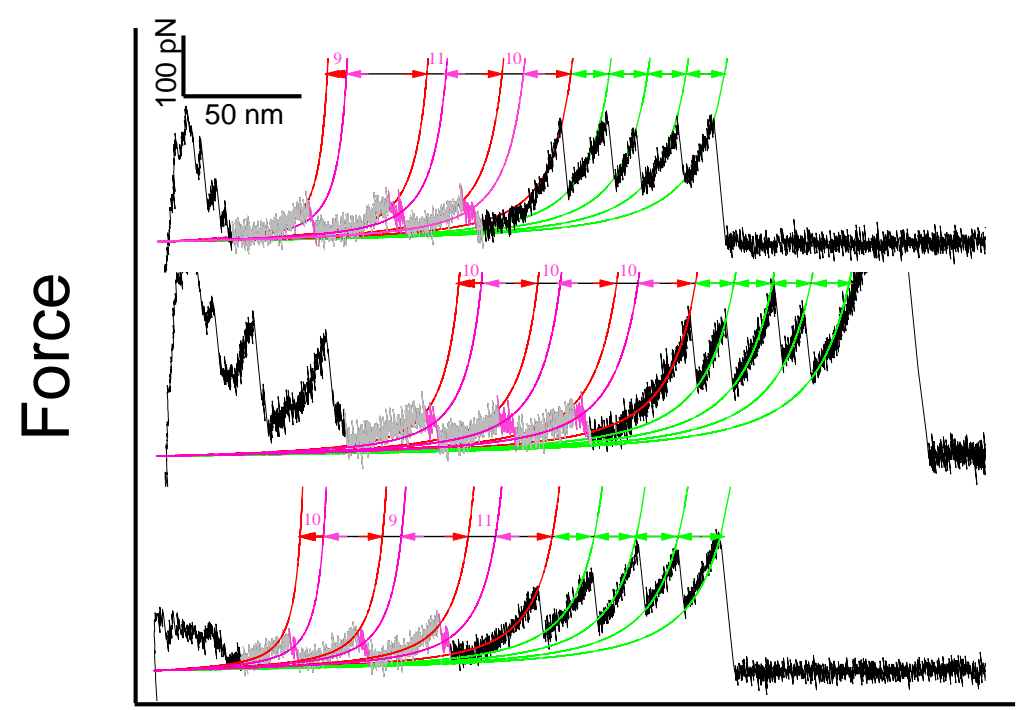

Extension 
Fig. S4 Some typical force-extension curves of (holo-azurin+GB1) 4 . The unfolding force peaks were fitting using the WLC model. The red lines correspond to the fitting curve of azurin, the pink lines correspond to the fitting curve of the copper cluster, and the green lines represent the fitting curves of GB1.

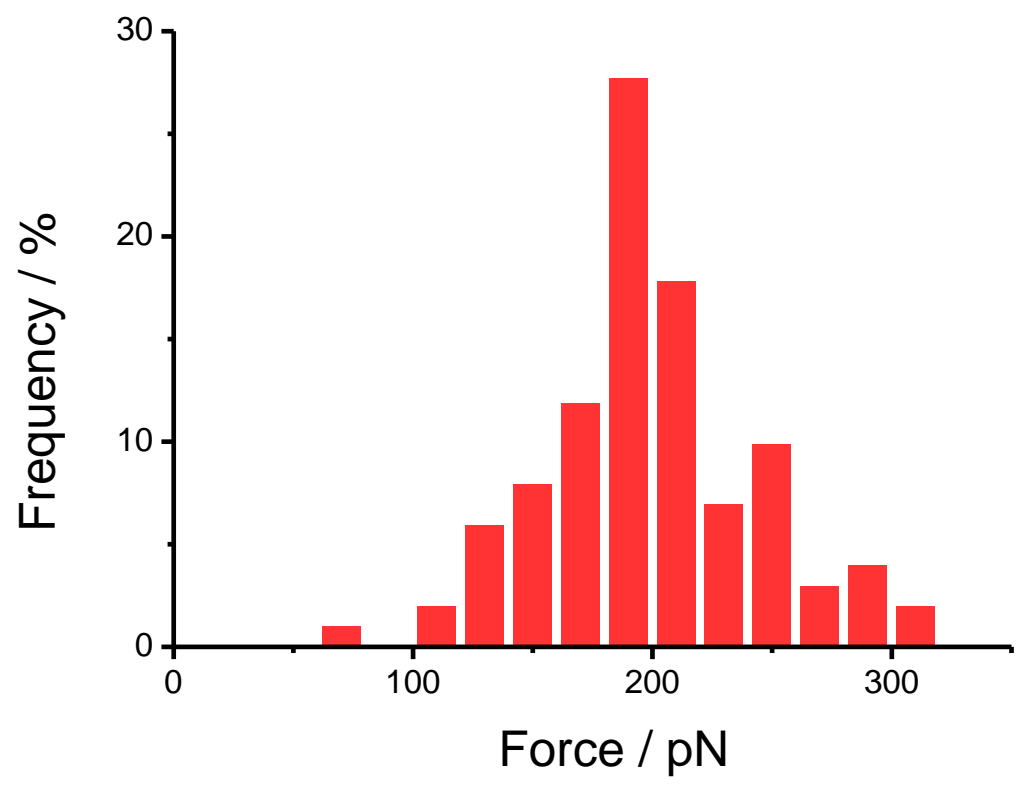

Fig. S5 Rupture force histogram for the rupture of the GB1 in the (apoazurin+GB1) 4 . The average unfolding force is $190.4 \pm 45.54 \mathrm{pN}$. 


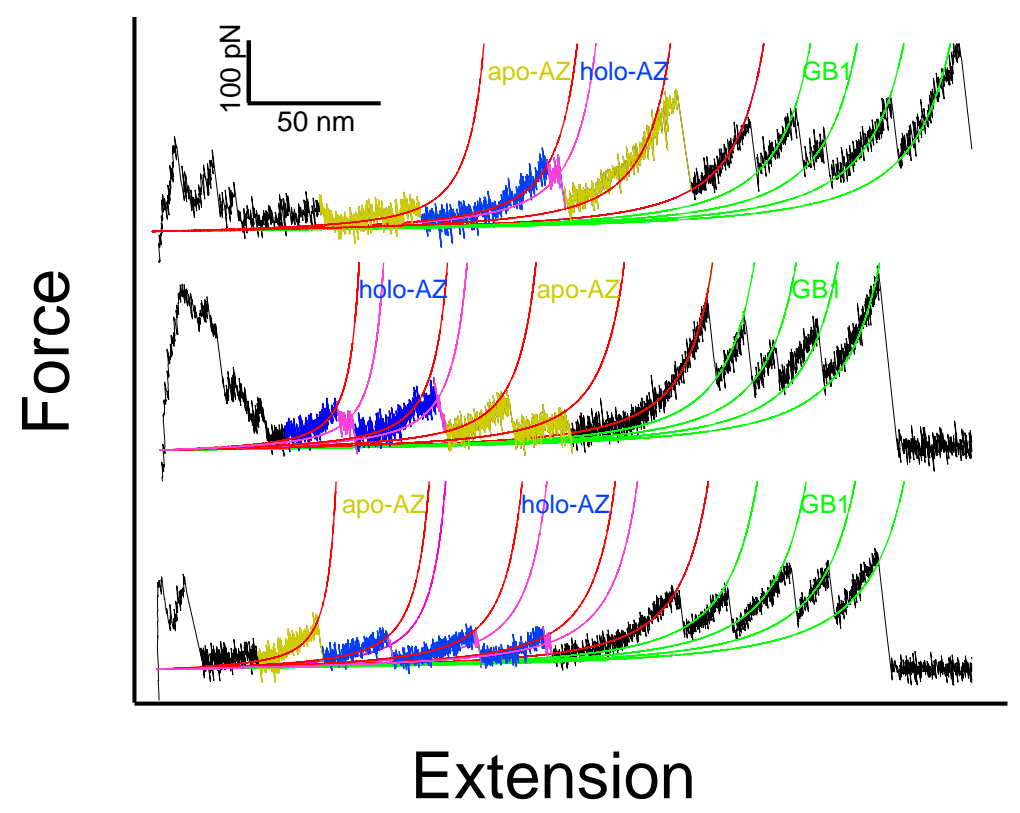

Fig. S6 Some typical force-extension curves of (holo-azurin+GB1) 4 with partial loss of copper cluster in azurin. The yellow curves respond to the unfolding of apo-azurin, the blue curves respond to the unfolding of holoazurin, pink curves respond to the unfolding of the copper cluster, and the black curves are the unfolding of GB1.

\section{Calculation of the number of cDNAs of the (azurin+GB1) 4}

The designed encoding cDNA of azurin (PDB 3JT2): the number of base pairs is 465 for this part.

GGATCCCATCATCACCATCACCATCATCATCACCATCACCATGCE-

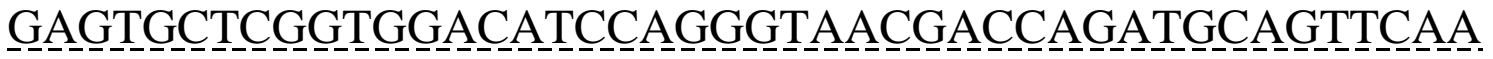

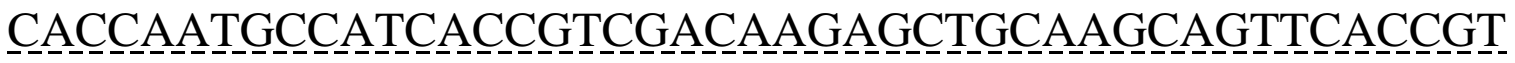
CAACCCTGTCCCAACCCCCGGCAACCCTGCCGAAAGAACGTCATIGGGCC

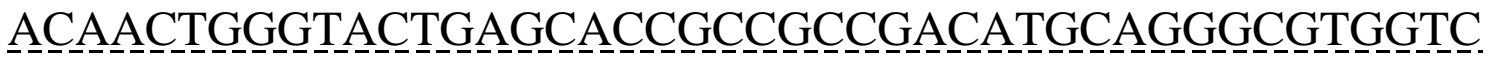

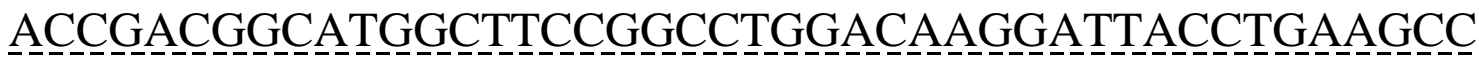

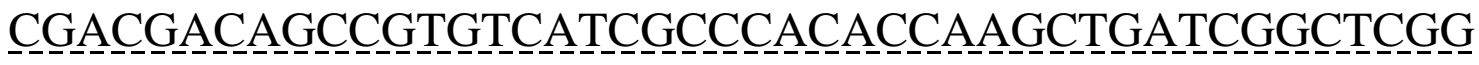
GCGAGAAGGACTCGGTGACCTTCGACGTCTCCAAGCTGA__GG__A_ 


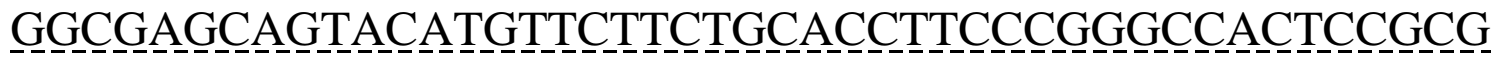

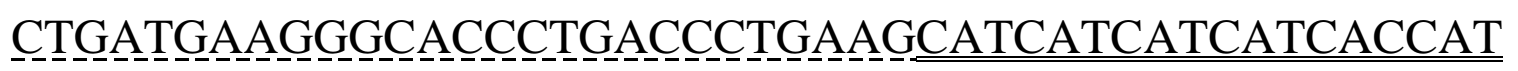

CACCATAGATCTGGCGGTACC

The designed encoding cDNA of GB1: the number of base pair is 252 for this part

GGATCCCATCATCACCATCACCATCATCATCACCATCACCATATG GACACCTACAAACTGATCCTGAACGGTAAAACCTGAAAGGTGA

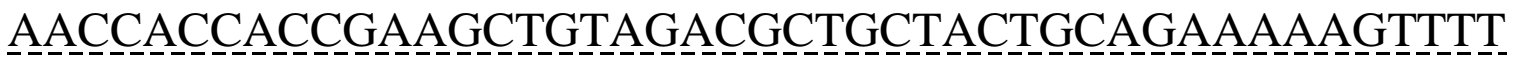

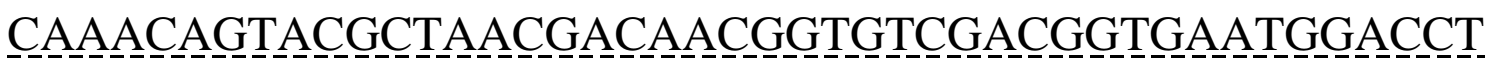
ACGACGACGCTACCAAAACCTTCACGGTTACCGAACATCATCATC ATCACCATCACCATAGATCTGGCGGTACC

The thick underline represents the BamHI site, the double underline presents the Histidine site, the wave underline represents the KpnI site, the dashed underlines are the interesting site.

Thus, the number of the designed cDNA of (azurin $+\mathrm{GB} 1)_{4}$ is $2868 \mathrm{bp}$ $((465+252) \times 4)$.

\section{Evaluation of the force needed to remove the copper ion from azurin can}

\section{be achieved by ultrasound treatment}

The force required to break the $\mathrm{Cu}-\mathrm{N}(\mathrm{His})$ bond is $80.1 \mathrm{pN}$, and the corresponding energy is $17.9 \pm 7.98 \mathrm{kcal} / \mathrm{mol}$ obtained by integrating the forceextension curves (Fig. 4A). It can be found that the force/energy needed to break the $\mathrm{Cu}-\mathrm{N}$ (His) bond is not high. Given to the fact that ultrasonic devices have been widely employed in bone cutting, dental, and spinal surgeries ${ }^{5}$, and the unfolding of proteins ${ }^{6,7}$. The energy needed for these applications of the ultrasonic devices is more higher (it takes about $4 \mathrm{kN}$ of force to break the typical human femur, $375 \mathrm{~J}$ of energy) than that for the break of $\mathrm{Cu}-\mathrm{N}(\mathrm{His})$. 
However, there are many factors (e.g., ultrasonic tip placement, size of sonoreactor, sample compositions, solutes size, solvent ratio,etc ${ }^{6}$ ) that should be considered during the measurements. Notably, the energy to break the $\mathrm{Cu}$ $\mathrm{N}$ (His) obtained in this study $(17.9 \pm 7.98 \mathrm{kcal} / \mathrm{mol})$ is comparable with that obtained by computer simulation $(19.7 \sim 24.4 \mathrm{kcal} / \mathrm{mol})^{8}$. These results further demonstrated that the AFM-based SMFS is an appropriate method to precisely obtain the force/energy needed to break a bond at the singlemolecule by experiment.

\section{Reference:}

1. Gronenborn A. M.; Filpula D. R.; Essig N. Z.; Achari A.; Whitlow M.; Wingfield P. T.; Clore G. M. A novel, highly stable fold of the immunoglobulin binding domain of streptococcal protein G Science (Washington DC) 1991, 253 (5020): 657-661. DOI: 10.1126/science. 1871600

2. Gronenborn AM; Filpula DR; Essig NZ; Achari A; Whitlow M; Wingfield PT; Clore GM. A novel, highly stable fold of the immunoglobulin binding domain of streptococcal protein G Science (Washington DC) 1991, 253 (5020): 657-661. DOI: 10.1126/science. 1871600

3. Cao Yi; Lam Canaan; Wang Meijia; Li Hongbin. Nonmechanical Protein Can Have Significant Mechanical Stability Angew. Chem. Int. Ed. 2006, 45 (4): 642-645. DOI: 10.1002/anie.200502623

4. Cao Yi; Li Hongbin. Polyprotein of GB1 is an ideal artificial elastomeric protein Nat. Mater. 2007, 6 (2): 109-114. DOI: 10.1038/nmat1825

5. Mae Tatsuo; Nakata Ken; Kumai Tsukasa; Ishibashi Yasuyuki; Suzuki Tomoyuki; Sakamoto Takamitsu; Ohori Tomoki; Hirose Takehito; Yoshikawa Hideki. Characteristics of ultrasound device: a new technology for bone curettage and excavation J. Orthop. Sci. 2019, 6 (1): 35. DOI: 10.1186/s40634-019-0203-7 
6. Rahman Md Mahfuzur; Lamsal Buddhi P. Ultrasound-assisted extraction and modification of plant-based proteins: Impact on physicochemical, functional, and nutritional properties Compr. Rev. Food Sci. F. 2021, 20 (2): 1457-1480. DOI: 10.1111/1541-4337.12709

7. Hu Hao; Wu Jiahui; Li-Chan Eunice C. Y.; Zhu Le; Zhang Fang; Xu Xiaoyun; Fan Gang; Wang Lufeng; Huang Xingjian; Pan Siyi. Effects of ultrasound on structural and physical properties of soy protein isolate (SPI) dispersions Food Hydrocolloids 2013, 30 (2): 647-655. DOI: 10.1016/j.foodhyd.2012.08.001

8. Pavelka Matěj; Burda Jaroslav V. Computational study of redox active centres of blue copper proteins: a computational DFT study Mol. Phys. 2008, 106 (24): 2733-2748. DOI: $10.1080 / 00268970802672684$ 